\title{
Effect of HOXA6 on the proliferation, apoptosis, migration and invasion of colorectal cancer cells
}

\author{
SHASHA WU ${ }^{1 *}$, FEIXIANG WU ${ }^{2 *}$ and ZHENG JIANG ${ }^{1}$ \\ Departments of ${ }^{1}$ Gastroenterology and ${ }^{2}$ Urology, \\ The First Affiliated Hospital of Chongqing Medical University, Chongqing 400016, P.R. China
}

Received December 1, 2017; Accepted March 27, 2018

DOI: $10.3892 /$ ijo.2018.4352

\begin{abstract}
Colorectal cancer (CRC) is one of the most common types of tumor worldwide. The morbidity and mortality rates of CRC have increased significantly in adults $<50$ years of age. In the present study, the effects of homeobox A6 (HOXA6) on the proliferation, apoptosis, migration and invasion of CRC cells were investigated. The results of reverse transcription-quantitative polymerase chain reaction (RT-qPCR) analysis demonstrated that the expression of HOXA6 in CRC tumor tissue was higher than that in adjacent normal tissue. Appropriate cell lines and plasmids were selected by RT-PCR and western blot analyses, and recombinant plasmids were transfected into $\mathrm{Caco} 2$ or HT-29 cells. The results of RT-qPCR and western blot analyses demonstrated that the expression of HOXA6 was effectively enhanced, or inhibited, following transfection. The rate of cell proliferation was measured with cell counting kit-8, colony formation assay and 5-ethynyl-2'-deoxyuridine assay, apoptosis was detected using terminal deoxynucleotidyl transferase dUTP nick end labeling and flow cytometry assays, and migration and invasion were evaluated using Transwell and wound-healing assays. The results demonstrated that the upregulation of HOXA6 promoted cell proliferation, migration and invasion, but inhibited apoptosis, whereas the downregulated expression of HOXA6 produced the opposite effects. In addition, the expression levels of apoptosis- and epithelial-mesenchymal transition (EMT)-related proteins were examined. The results of the western blot analysis revealed that the upregulated expression of HOXA6 suppressed the expression of
\end{abstract}

Correspondence to: Dr Zheng Jiang, Department of Gastroenterology, The First Affiliated Hospital of Chongqing Medical University, 1 Youyi Road, Chongqing 400016, P.R. China

E-mail: jiangz1753@163.com

${ }^{*}$ Contributed equally

Abbreviations: CRC, colorectal cancer; CCK-8, cell counting kit-8; RT-qPCR, reverse transcription-quantitative polymerase chain reaction; EMT, epithelial-mesenchymal transition

Key words: homeobox A6, colorectal cancer, cell proliferation, apoptosis, invasion
B-cell lymphoma-2 (Bcl-2)-associated X protein, caspase-3, poly(ADP-ribose) polymerase and E-cadherin, but promoted the expression of $\mathrm{Bcl}-2, \mathrm{~N}$-cadherin and Vimentin, whereas the opposite effect was observed in cells with downregulated HOXA6. These results indicated that HOXA6 regulated apoptosis through the $\mathrm{Bcl}-2$ signaling pathway, and regulated migration and invasion through the EMT process. In conclusion, the present study confirmed that HOXA6 was involved in the regulation of $\mathrm{CRC}$, which may inform the development of strategies for the diagnosis and treatment of CRC.

\section{Introduction}

Colorectal cancer (CRC) is one of the leading causes of cancer-associated mortality worldwide. Although the overall morbidity and mortality rates for CRC have been declining for decades, they have increased considerably in adults $<50$ years of age. In addition, the 5-year relative survival rate for patients with CRC diagnosed at an advanced stage is just $14 \%$, as not all of the tumor can be surgically removed (1-3). In addition to old age and lifestyle factors, genetic changes are also a cause of CRC (4). In these cases of CRC, targeted therapy may be effective. Therefore, the identification of novel tumor markers may contribute to the diagnosis and treatment of CRC, such as the effect of the clinical application of carcinoembryonic antigen and carbohydrate antigen 19-9 (5).

The homeobox (HOX) family includes a series of developmental genes. The encoded homeodomain proteins are important transcription factors that bind to specific DNA sequences as monomers or multimers to regulate cell differentiation, proliferation and apoptosis. At present, 39 human HOX genes have been identified, which are divided into four clusters: A, B, C and D. Previous studies have reported that HOX family genes are involved in the development of human malignancies and are associated with prognosis $(6,7)$. It was previously observed that HOXA9, HOXA13 and HOXB7 were overexpressed in esophageal cancer, and promoted the proliferation of esophageal cancer cells (8-10). The expression levels of HOXA1, HOXA10, HOXA13, HOXB7 and HOXC6 were also found to be increased in gastric cancer tissue, and may promote cell proliferation and metastasis, in addition to being associated with prognosis (11-17). In CRC, HOXB7 was also reported to be expressed at a high level, and interacted with the mitogen-activated protein kinase and 
phosphoinositide 3-kinase/AKT pathways to promote tumor cell proliferation (18).

HOXA6 is a member of the HOX family and encodes a DNA-binding transcription factor, which may regulate gene expression, morphogenesis and differentiation. Previous studies have reported that the HOXA6 gene is expressed at a high level in several types of malignant tumor, including cerebral glioma and acute myeloid leukemia, and that it is associated with cell invasion, proliferation, colony formation and chemosensitivity $(19,20)$. HOX family members have been confirmed to be involved in tumor regulation, including in the occurrence and development of CRC. As HOXA6 is also involved in the regulation of certain types of malignant tumor, it is reasonable to suggest that HOXA6 may have a similar effect on tumor regulation in CRC. Based on this, the aim of the present study was to investigate the expression of HOXA6 in $\mathrm{CRC}$, and the effect of the expression of HOXA6 on the proliferation, apoptosis, migration and invasion of CRC cells.

\section{Materials and methods}

Patient specimens. A total of $16 \mathrm{CRC}$ tumor and paired adjacent normal colorectal tissue samples were collected from the First Affiliated Hospital of Chongqing Medical University (Chongqing, China) between September 28, 2017 and November 11, 2017 (Table I). Informed consent was signed by all patients, and ethics approval was obtained from the Ethics Committee of the First Affiliated Hospital of Chongqing Medical University.

Cell culture and transfection. The Caco2, HCT116, SW480 and HT-29 human CRC cell lines were purchased from the American Type Culture Collection (Manassas, VA, USA) and cultured in RPMI-1640 medium (HyClone/GE Healthcare Life Sciences, Logan, UT, USA) supplemented with $10 \%$ fetal bovine serum (PAN-Biotech $\mathrm{GmbH}$, Aidenbach, Germany) at $37^{\circ} \mathrm{C}$ in $5 \% \mathrm{CO}_{2}$. Plasmids expressing HOXA6, short hairpin interfering RNA against HOXA6 (shHOXA6; sh1, 5'-CCTTGT TTCTACCAACAGTCC-3'; sh2, 5'-CCTCGTGTTTCTATT CTGATA-3'; sh3, 5'-GGCGCGCAAATGAGTTCCTAT-3'; sh4, 5'-GACAAGACGTACACCTCACCT-3') (21) or a negative control (NC) sequence (non-targeting shRNA) were purchased from GeneCopeia, Inc. (Rockville, MD, USA). The CRC cells were transfected using Lipofectamine 2000 (Invitrogen; Thermo Fisher Scientific, Inc., Waltham, MA, USA) according to the manufacturer's protocol. For the selection of stable cell lines, G418 and Puromycin (Sigma-Aldrich; Merck Millipore, Darmstadt, Germany) were added to the medium at $48 \mathrm{~h}$ following transfection and the cells were maintained under the aforementioned conditions.

Reverse transcription-polymerase chain reaction ( $R T-P C R)$ and reverse transcription-quantitative PCR (RT-qPCR) analyses. Total RNA was extracted from tissue samples and CRC cells using TRIzol reagent (Invitrogen; Thermo Fisher Scientific, Inc.). RNA was reverse transcribed into cDNA using the GoScript $^{\mathrm{TM}}$ Reverse Transcription system (Promega Corp., Madison, WI, USA). All PCR primers were purchased from Genscript (Nanjing, China): HOXA6 forward, 5'-TACACG CGCTACCAGACAC-3' and reverse, 5'-GCGTGGAATTGAT GAGCTTGTTT-3' (product length, $178 \mathrm{bp}$ ); $\beta$-actin forward,
Table I. Characteristics of the patients with colorectal cancer.

\begin{tabular}{|c|c|c|c|}
\hline $\begin{array}{l}\text { Age } \\
\text { (years) }\end{array}$ & Sex & Diagnosis & $\begin{array}{l}\text { Sample collection date } \\
\text { (all in the year 2017) }\end{array}$ \\
\hline 74 & Male & Colon carcinoma & September 28 \\
\hline 72 & Male & Rectal carcinoma & September 30 \\
\hline 67 & Male & Rectal carcinoma & October 9 \\
\hline 63 & Female & Rectal carcinoma & October 11 \\
\hline 63 & Male & Colon carcinoma & October 13 \\
\hline 68 & Male & Rectal carcinoma & October 13 \\
\hline 65 & Female & Rectal carcinoma & October 14 \\
\hline 62 & Female & Colon carcinoma & October 17 \\
\hline 66 & Female & Colon carcinoma & October 20 \\
\hline 74 & Male & Colon carcinoma & October 21 \\
\hline 63 & Male & Colon carcinoma & October 25 \\
\hline 80 & Female & Colon carcinoma & October 25 \\
\hline 69 & Male & Colon carcinoma & October 31 \\
\hline 65 & Female & Colon carcinoma & November 6 \\
\hline 49 & Male & Colon carcinoma & November 9 \\
\hline 63 & Male & Colon carcinoma & November 10 \\
\hline
\end{tabular}

5'-TGGAACGGTGAAGGTGACAG-3' and reverse, 5'-AAC AACGCATCTCATATTTGGAA-3' (product length, 125 bp). RT-qPCR was performed with GoTaq qPCR Master mix (Promega Corporation) (nuclease-free water: $3.6 \mu \mathrm{l}$; upstream primer: $0.2 \mu \mathrm{l}$; downstream primer: $0.2 \mu \mathrm{l} ; 2 \mathrm{X}$ GoTaq ${ }^{\circledR} \mathrm{qPCR}$ Master Mix: $5 \mu \mathrm{l}$; cDNA: $1 \mu \mathrm{l}$. Thermocycling steps: $95^{\circ} \mathrm{C}-10 \mathrm{~min} ; 95^{\circ} \mathrm{C}-15 \mathrm{sec} ; 60^{\circ} \mathrm{C}-1 \mathrm{~min}, 40$ cycles) and the data were analyzed using the $2^{-\Delta \Delta \mathrm{Cq}}$ method (22). RT-PCR was performed with GoTaq polymerase (Promega Corporation) according to the manufacturer's protocol (2X GoTaq Green Master Mix: $5 \mu \mathrm{l}$; upstream primer: $0.6 \mu \mathrm{l}$; downstream primer: $0.6 \mu \mathrm{l}$; cDNA: $2 \mu \mathrm{l}$; nuclease-free water: $1.8 \mu \mathrm{l}$. Thermocycling steps: $95^{\circ} \mathrm{C}-2 \mathrm{~min} ; 95^{\circ} \mathrm{C}-30 \mathrm{sec}, 55^{\circ} \mathrm{C}-30 \mathrm{sec}, 72^{\circ} \mathrm{C}-30 \mathrm{sec}$; $72^{\circ} \mathrm{C}-3$ min. HOXA6: 32 cycles, $\beta$-actin: 23 cycles.). The PCR products were analyzed with $2 \%$ agarose gel electrophoresis, and subjected to densitometric analysis with a gel imaging system (Bio-Rad Laboratories, Inc., Hercules, CA, USA).

Western blot analysis. Proteins were extracted from the cells at $72 \mathrm{~h}$ post-transfection using RIPA lysis buffer (Beyotime Institute of Biotechnology, Haimen, China). The protein concentration was determined using a BCA kit (Beyotime Institute of Biotechnology). Subsequent to $10 \%$ SDS-PAGE separation $(40 \mu \mathrm{g})$, the proteins were transferred onto a PVDF membrane. The membrane was blocked in 5\% skim milk at room temperature for $1-2 \mathrm{~h}$, and then incubated at $4^{\circ} \mathrm{C}$ overnight with the primary antibodies. Following incubation with appropriate horseradish peroxidase-conjugated antirabbit secondary antibodies (\#7074, dilution: 1:3,000) (Cell Signaling Technology, Inc., Danvers, MA, USA) for $2 \mathrm{~h}$ at room temperature, the membranes were visualized with an ECL kit (Beyotime Institute of Biotechnology). The grayscale value of images was analyzed using Fusion software (Fusion FX, Vilber lourmat). The primary antibodies for western blot analysis included rabbit anti-HOXA6 (YT2212, 1:800) and 
anti- $\beta$-actin (A283, 1:1,000) from ImmunoWay Biotechnology Company (Plano, TX, USA), and rabbit anti-B-cell lymphoma-2 (Bcl-2, \#3498, 1:3,000), anti-Bcl-2-associated X protein (Bax, \#5023, 1:3,000), anti-cleaved-caspase-3 (\#9662, 1:1,000), anti-poly (ADP-ribose) polymerase (PARP, \#9532, 1:3,000), anti-N-cadherin (\#13116, 1:3,000), anti-E-cadherin (\#3195, 1:3,000) and anti-Vimentin (\#5741, 1:3,000) from Cell Signaling Technology, Inc.

Cell proliferation assay. Cell proliferation was assessed using a cell counting kit-8 (CCK-8) assay. The stably transfected cells, including the Caco2-HOXA6, Caco2-NC, HT-29-shHOXA6 and HT-29-NC cells, were seeded in a 96-well plate at a density of $4 \times 10^{3}$ cells/well, and cultured overnight. CCK- 8 reagent was then added into the wells and the absorbance at $450 \mathrm{~nm}$ was detected following culture for 24,48 and $72 \mathrm{~h}$.

For the colony formation assay, the stably transfected cells were seeded in a 6-well plate at a density of 500 cells/well. Following culture for 2 weeks, the clones were fixed with paraformaldehyde and stained with gentian violet. The numbers of clones containing $>50$ cells were counted under an inverted phase contrast microscope (Leica, Wetzlar, Germany).

A 5-ethynyl-2'-deoxyuridine (EdU) assay was also performed to measure cell proliferation ability. An EdU kit (RiboBio Co., Ltd., Guangzhou, China) was used according to the manufacturer's protocol. Images were captured with a fluorescence microscope (Olympus Corporation, Tokyo, Japan).

Cell migration and invasion. Cell migration and invasion were measured using Transwell assays. To determine the rate of cell migration, $200 \mu \mathrm{l}$ of RPMI-1640 medium containing $5 \times 10^{4}$ cells was added into the upper chamber, and $700 \mu 1$ RPMI-1640 containing 10\% FBS was added into the lower chamber. Following incubation for $48 \mathrm{~h}$, the migrated cells were fixed and stained with crystal violet for $15 \mathrm{~min}$. Subsequently, the cells were viewed and counted under an inverted phase contrast microscope in five fields of view. The invasion ability was measured with Matrigel (BD Biosciences, Franklin Lakes, NJ, USA). The Matrigel was diluted with RPMI-1640 and added to the upper chamber (100 $\mu \mathrm{l} /$ well). Following solidification of the Matrigel, $100 \mu \mathrm{l}$ of RPMI-1640 containing $1 \times 10^{5}$ cells was added into the upper chamber; the remaining steps were the same as for the migration assay.

Cell migration was also detected using a wound-healing assay. The stably transfected cells were seeded in a 6-well plate. At confluence, the cell monolayer was scratched, washed with PBS, and maintained in serum-free RPMI-1640. Following incubation for 0,24 and $48 \mathrm{~h}$, images were captured using an inverted microscope.

Cell apoptosis. Flow cytometry was used to detect the rate of apoptosis of $\mathrm{Caco} 2$ and HT-29 cells. At 48 h posttransfection, the cells were harvested with EDTA-free trypsin and suspended in PBS buffer. The cells were stained with an Annexin V-FITC/PI kit (BD Biosciences) according to the manufacturer's protocol, and immediately analyzed by flow cytometry.

Apoptosis was also measured using a terminal deoxynucleotidyl transferase dUTP nick end labeling (TUNEL) assay kit (Roche Applied Science, Indianapolis, IN, USA), according to the manufacturer's protocol. Following TUNEL staining, the cells were stained with DAPI for $5 \mathrm{~min}$ and washed with PBS. A fluorescence microscope (Leica) was used to capture images.

Statistical analysis. Each experiment was independently repeated three times. Statistical analysis was performed with SPSS version 22 (IBM SPSS, Armonk, NY, USA). All data are presented as the mean \pm standard deviation and were analyzed using Student's t-test (two groups) or two-way analysis of variance [for the results of CCK-8 assay (Fig. 2A) and wound-healing assay (Fig. 4B)] followed by Bonferroni's test. A value of $\mathrm{P}<0.05$ was considered to indicate a statistically significant difference.

\section{Results}

Expression of HOXA6 in human CRC tissues and cell lines. The detection of the mRNA expression of HOXA6 in CRC and normal colorectal tissue samples was performed by RT-qPCR analysis. The results showed that the mRNA levels of HOXA6 in the CRC tissues were significantly upregulated, compared with those in the paired normal colorectal tissue samples $(\mathrm{P}=0.0103)$ (Fig. 1A). Subsequently, the expression levels of HOXA6 in four CRC cell lines (Caco2, SW480, HCT116 and HT-29) were detected by RT-PCR analysis. The mRNA expression level of HOXA6 was highest in the HT-29 cells and lowest in Caco 2 cells (Fig. 1B). Therefore, these two cell lines were selected for the subsequent experiments.

Verification of the knockdown efficiency of the shHOXA6 plasmid. To analyze the knockdown efficiency of four interference plasmids targeting HOXA6 (shRNA1/2/3/4-HOXA6), the expression of HOXA6 in transfected cells was examined by RT-PCR and western blot analyses. HOXA6 was expressed the least in the cells transfected with shRNA1-HOXA6, as confirmed by RT-PCR and western blot analyses (Fig. 1B). Based on these results, shRNA1-HOXA6 was selected as the most efficient interference plasmid for the remaining experiments.

Verification of the expression of HOXA6 in transfected cells. The Caco 2 cells were transfected with plasmids expressing HOXA6 or an NC and HT-29 cells were transfected with shHOXA6 or NC plasmids. The expression of HOXA6 was detected by RT-qPCR and western blot analyses. The results showed that the mRNA and protein expression levels were significantly upregulated in the Caco2-HOXA6 cells $(\mathrm{P}=0.0008$ and 0.0012) and suppressed in the HT-29-shHOXA6 cells ( $\mathrm{P}=0.0225$ and 0.0026$)$ (Fig. $1 \mathrm{C}$ and $\mathrm{D})$.

HOXA6 enhances the proliferation rate of $C R C$ cells. To examine the effect of HOXA6 on CRC cell proliferation, CCK-8, colony formation and EdU assays were performed. The CCK-8 assay demonstrated that the Caco2-HOXA6 cells grew significantly faster than the Caco2-NC cells $(\mathrm{P}<0.0001)$, whereas the growth rate of the HT-29-shHOXA6 cells was significantly lower, compared with that of the HT-29-NC cells $(\mathrm{P}<0.0001)$ (Fig. $2 \mathrm{~A})$. In addition, the colony formation 
A
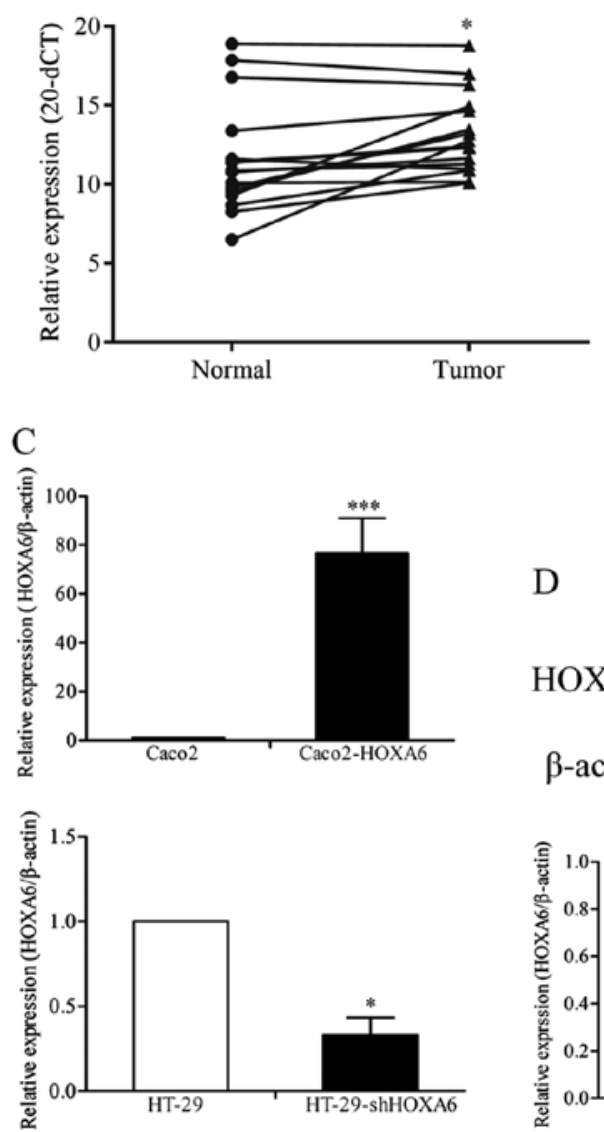

$\mathrm{D}$
B

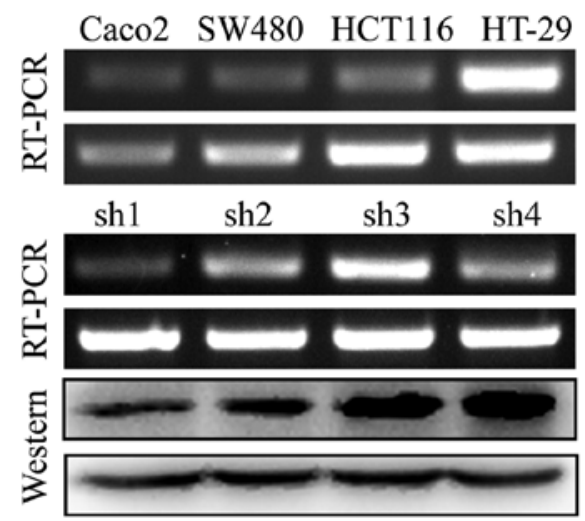

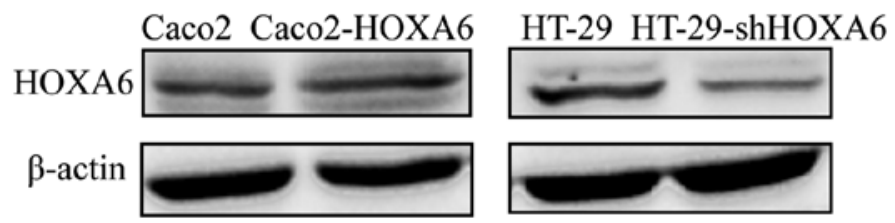
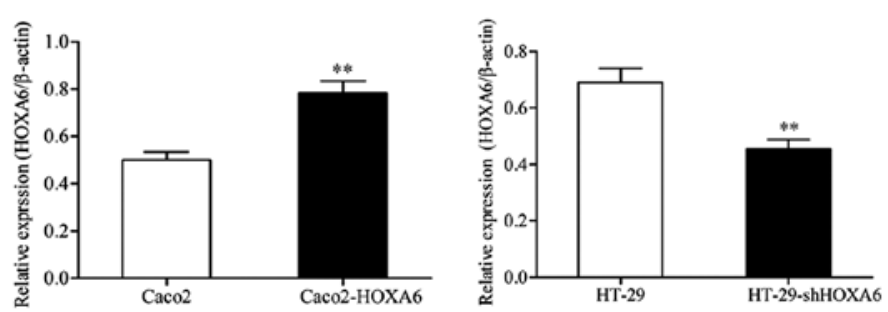

Figure 1. Expression of HOXA6 in CRC tissues, cell lines and transfected HT-29 cells. (A) Relative mRNA expression of HOXA6 in 16 paired CRC tumor and normal adjacent tissues was detected by RT-qPCR and normalized against $\beta$-actin. The data were analyzed using a paired t-test. (B) mRNA expression of HOXA6 in four CRC cell lines as demonstrated by RT-PCR analysis, and in HT-29 cells transfected with four interference plasmids as demonstrated by RT-PCR and western blot analyses. (C) Determination of the overexpression or inhibition efficiency of plasmid transfection by RT-qPCR analysis. (D) Western blot analysis results for protein expression of HOXA6 in the transfected cells. Three independent experiments were performed for each assay. ${ }^{*} \mathrm{P}<0.05,{ }^{* *} \mathrm{P}<0.01$ and ${ }^{* * *} \mathrm{P}<0.001$. HOXA6, homeobox A6; CRC, colorectal cancer; sh, short hairpin RNA; RT-qPCR, reverse transcription-quantitative polymerase chain reaction; RT-PCR, reverse transcription-PCR.

assay demonstrated that the Caco2-HOXA6 cells formed more colonies than the Caco2-NC cells $(\mathrm{P}<0.0001)$, whereas the HT-29-shHOXA6 cells formed fewer colonies than the HT-29-NC cells ( $\mathrm{P}=0.0004)$ (Fig. 2B). The EdU assay demonstrated that the upregulated expression of HOXA6 promoted cell proliferation $(\mathrm{P}=0.0301)$, whereas the downregulation of HOXA6 suppressed cell proliferation $(\mathrm{P}=0.0310)$ (Fig. 2C). These results indicated that the expression of HOXA6 enhanced the proliferative capacity of the CRC cells.

HOXA6 inhibits apoptosis in CRC cells. The effect of HOXA6 on CRC cell apoptosis was evaluated with flow cytometry and a TUNEL assay. The results of the two assays revealed that the rate of apoptosis of the Caco2-HOXA6 cells was inhibited, compared with that of the Caco2-NC cells $(\mathrm{P}<0.05)$, whereas the rate of apoptosis of the HT-29-shRNA cells was significantly higher, compared with that of the HT-29-NC cells $(\mathrm{P}<0.01)$ (Fig. 3A and $\mathrm{B})$. Western blot analysis was performed to detect the protein expression levels of PARP, Bcl-2, Bax and caspase-3. The protein expression levels of cleaved PARP, Bax and cleaved caspase-3 were inhibited in the Caco2-HOXA6 cells and increased in the HT-29-shRNA cells, whereas the expression of Bcl-2 was increased in the
Caco2-HOXA6 cells and suppressed in the HT-29-shHOXA6 cells, compared with levels in the NC cells (Fig. 3C). These results demonstrated that HOXA6 inhibited the apoptosis of the CRC cells.

Expression of HOXA6 enhances CRC cell migration and invasion. Transwell and wound-healing assays were performed to analyze the effect of HOXA6 on CRC cell migration and invasion. The results of the Transwell assay showed that the numbers of migrated and invaded Caco2-HOXA6 cells were significantly increased, whereas the numbers of migrated and invaded HT-29-shHOXA6 cells were suppressed, compared with those in the NC (Fig. 4A). The wound-healing assay demonstrated that the Caco2-HOXA6 cells migrated at a faster rate than the Caco2-NC cells $(\mathrm{P}=0.0186)$, whereas the HT-29-shHOXA6 cells migrated at a slower rate than the HT-29-NC cells ( $\mathrm{P}=0.0003)$ (Fig. 4B). The results of the western blot analysis showed that the expression levels of $\mathrm{N}$-cadherin and Vimentin increased, whereas the expression of E-cadherin decreased in the Caco2-HOXA6 cells, with the opposite pattern observed in the HT-29-shHOXA6 cells (Fig. 4C). These results confirmed that HOXA6 enhanced the migration and invasion of the CRC cells. 
A
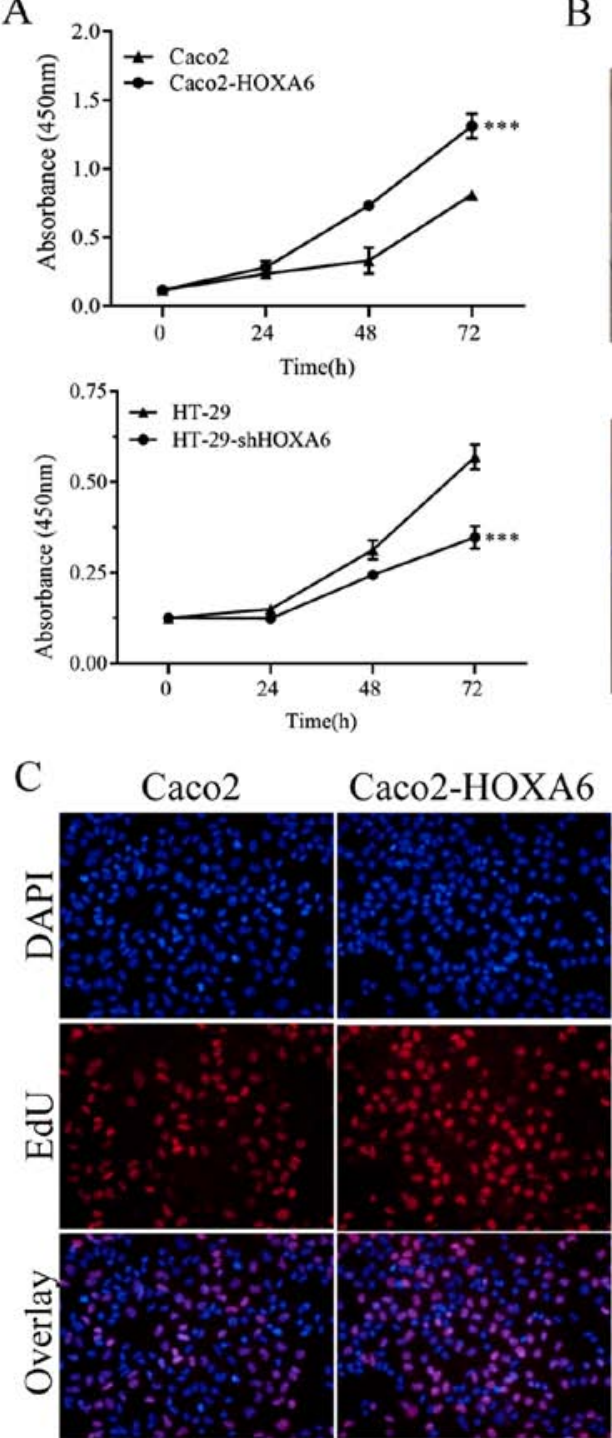

B
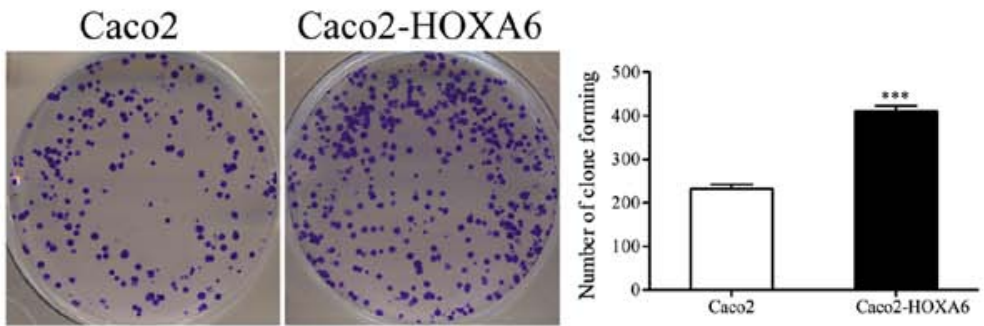

HT-29
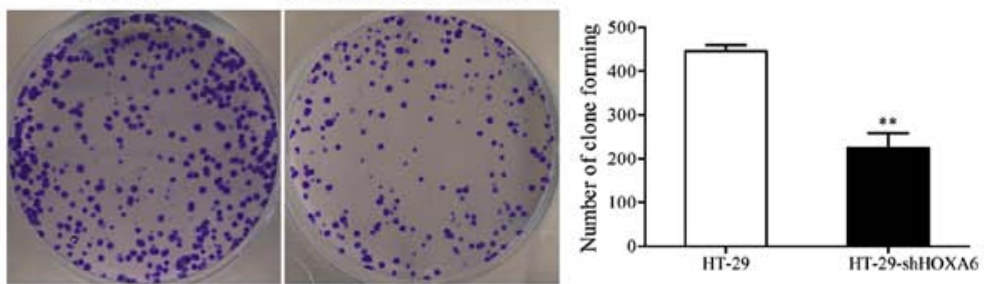

Figure 2. Cell proliferation measured with CCK-8, colony formation and EdU staining assays. (A) CCK-8 results for the transfected cells. (B) Colony formation results for the transfected cells. (C) EdU staining results for the transfected cells (x200 magnification). Cells stained with DAPI and EdU were considered as positive cells. Three independent experiments were performed for each assay. ${ }^{*} \mathrm{P}<0.05,{ }^{* *} \mathrm{P}<0.01$ and ${ }^{* * * *} \mathrm{P}<0.001$. CCK-8, cell counting kit-8; EdU, 5-ethynyl-2'-deoxyuridine; sh, short hairpin RNA; HOXA6, homeobox A6.

\section{Discussion}

The occurrence and development of tumors are associated with several factors, including behavioral and environmental factors. The occurrence of tumors is the result of the accumulation of mutations in a number of genes over an extended period of time. The identification of affected genes may provide a basis for the early diagnosis and treatment of cancer.

HOXA6, a member of the HOX family, has been reported to be involved in the regulation of certain types of malignancy. The present study investigated the effect of the expression of HOXA6 on CRC cell proliferation, apoptosis, migration and invasion, and examined the expression of associated proteins. Initially, the RT-qPCR results demonstrated that HOXA6 was upregulated in CRC samples compared with normal tissues. Subsequently, by upregulating and downregulating the expression of HOXA6 with plasmid transfection, it was found that the upregulated expression of HOXA6 promoted proliferation, migration and invasion, and inhibited apoptosis, whereas the downregulated expression of HOXA6 had the opposite effects. Finally, the expression levels of associated proteins were detected. The upregulated expression of HOXA6 increased the expression levels of cleaved PARP, cleaved caspase-3, Bax, $\mathrm{N}$-cadherin and Vimentin, but decreased the expression levels of Bcl-2 and E-cadherin. The downregulated expression of HOXA6 induced the opposite results. These results suggested that HOXA6 promoted proliferation and metastasis, and inhibited apoptosis in CRC.

Apoptosis is the process of programmed cell death, which eliminates unnecessary or unhealthy cells from the body. Cancer cells avoid apoptosis, and gain an advantage in survival and proliferation by promoting anti-apoptotic mechanisms and downregulating pro-apoptotic programs (23). Apoptosis is regulated by the Bcl-2 and caspase families, particularly caspase-3. The activation of caspase- 3 is necessary for efficient apoptosis (24). The balance between pro-apoptotic and antiapoptotic Bcl-2 family proteins is also significant in apoptosis. Previous studies have identified that the $\mathrm{Bcl}-2 / \mathrm{Bax}$ ratio was 
A
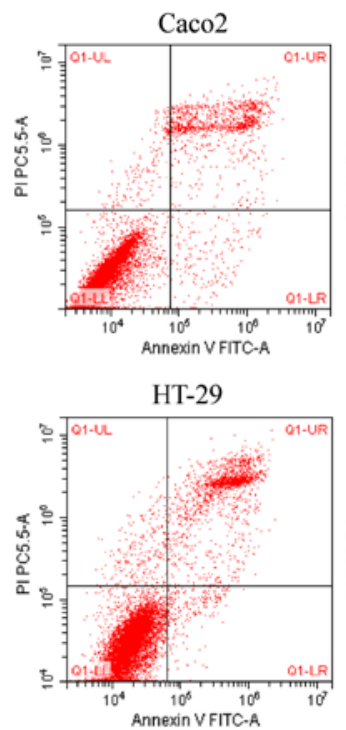
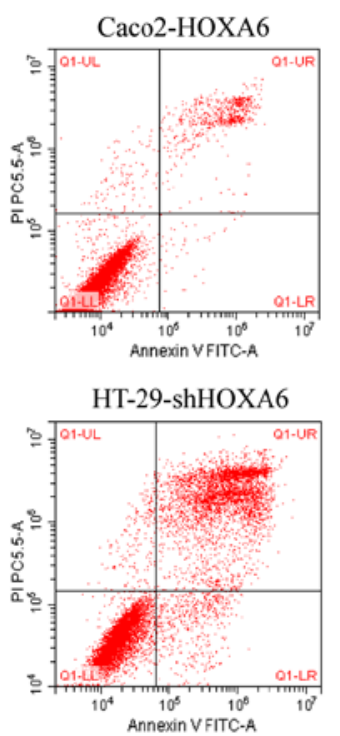
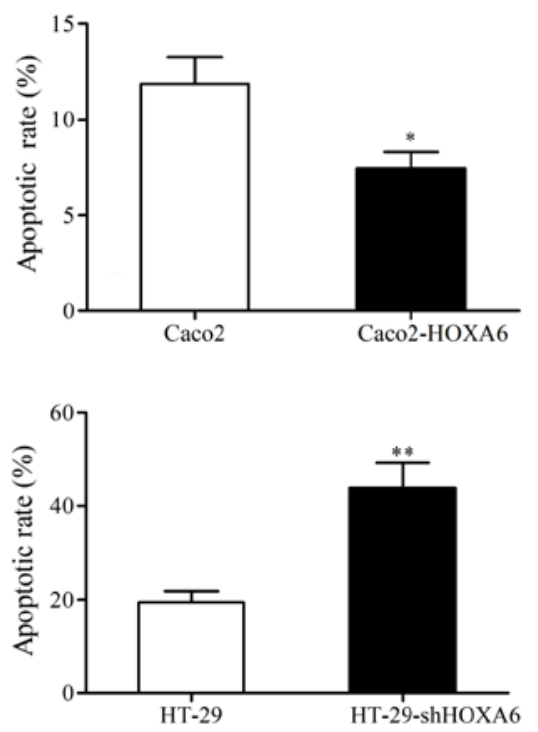

B

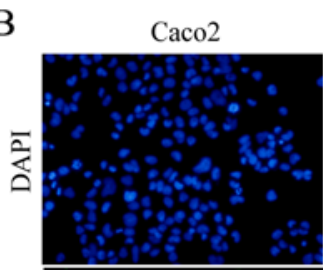

Caco2-HOXA6

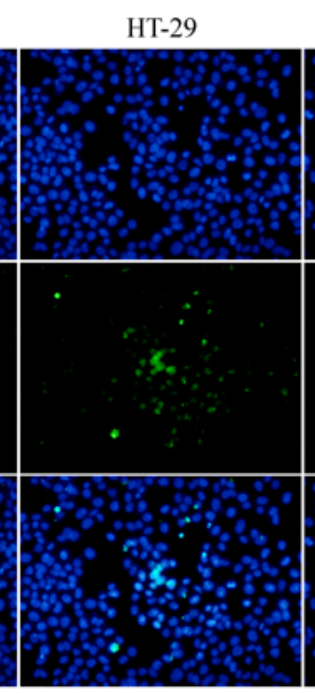

HT-29-shHOXA6
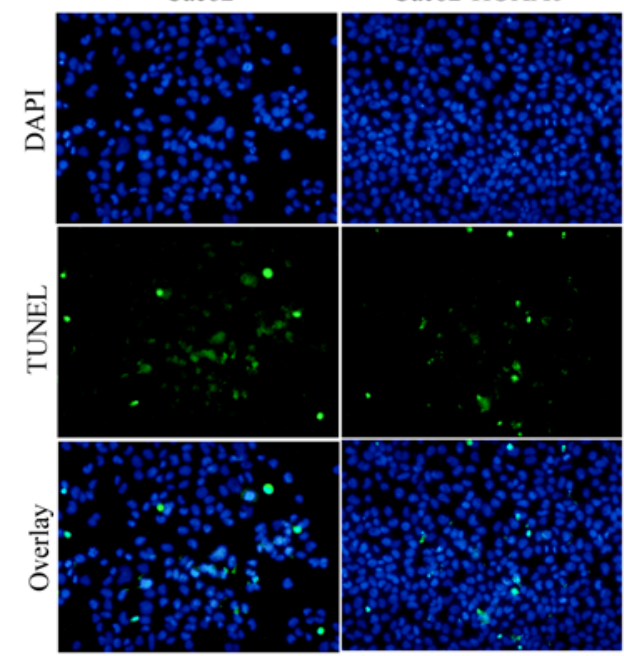

$\mathrm{C}$
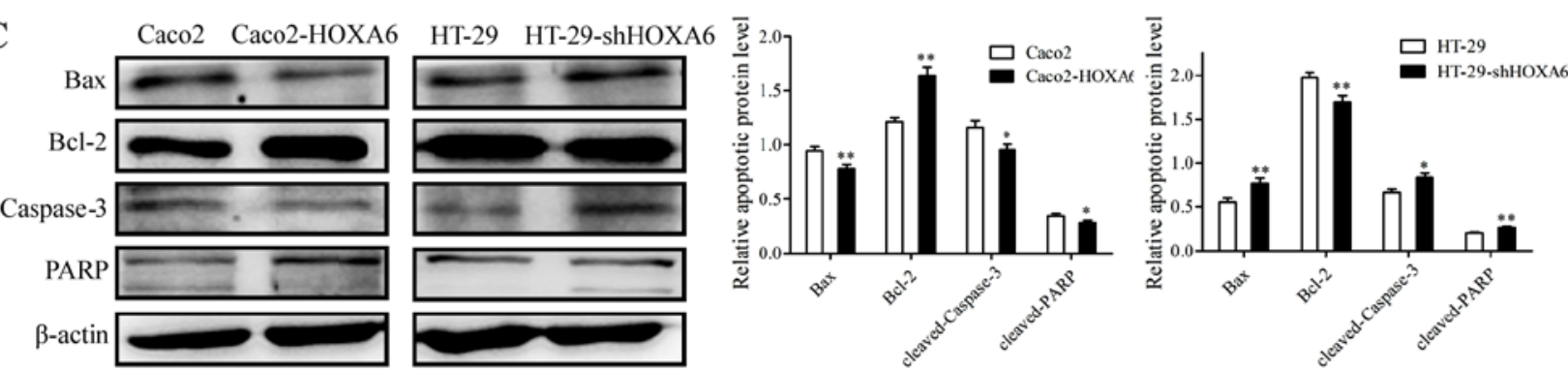

Figure 3. Rate of apoptosis in transfected cells, as detected by flow cytometry and TUNEL assays, and the expression of apoptosis-related proteins. (A) Results of flow cytometry for the rate of apoptosis in the transfected cells. (B) Results of the TUNEL assay, which also measured the rate of apoptosis (x200 magnification). Cells stained with both TUNEL and DAPI were considered as apoptotic cells. (C) Western blot analysis to detect the protein expression levels of Bax, Bcl-2, caspase-3 and PARP. Three independent experiments were performed for each assay. ${ }^{*} \mathrm{P}<0.05$ and ${ }^{* *} \mathrm{P}<0.01$. TUNEL, terminal deoxynucleotidyl transferase dUTP nick end labeling; Bcl-2, B-cell lymphoma 2; Bax, Bcl-2-assocated X protein; PARP, poly(ADP-ribose) polymerase; sh, short hairpin RNA; HOXA6, homeobox A6.

increased in tumor tissues, causing a reduced rate of apoptosis $(25,26)$. PARP is cleaved by caspase-3 during apoptosis, which means that PARP cleavage is an important indicator of apoptosis and caspase-3 activation $(27,28)$. This is consistent with the experimental results of the present study described above.

Tumor metastasis is the main cause of the poor prognosis of CRC. Following the determination of changes in migration and invasion, the expression of epithelial-mesenchymal transition (EMT)-related proteins was detected. It has been demonstrated that EMT initiates the isolation of cancer cells from primary carcinomas, which subsequently migrate and spread further $(29,30)$. Its core features are a decreased expression of E-cadherin and increased expression of Vimentin. The experimental results in the present study confirmed that an increase in the expression of HOXA6 promoted EMT. 

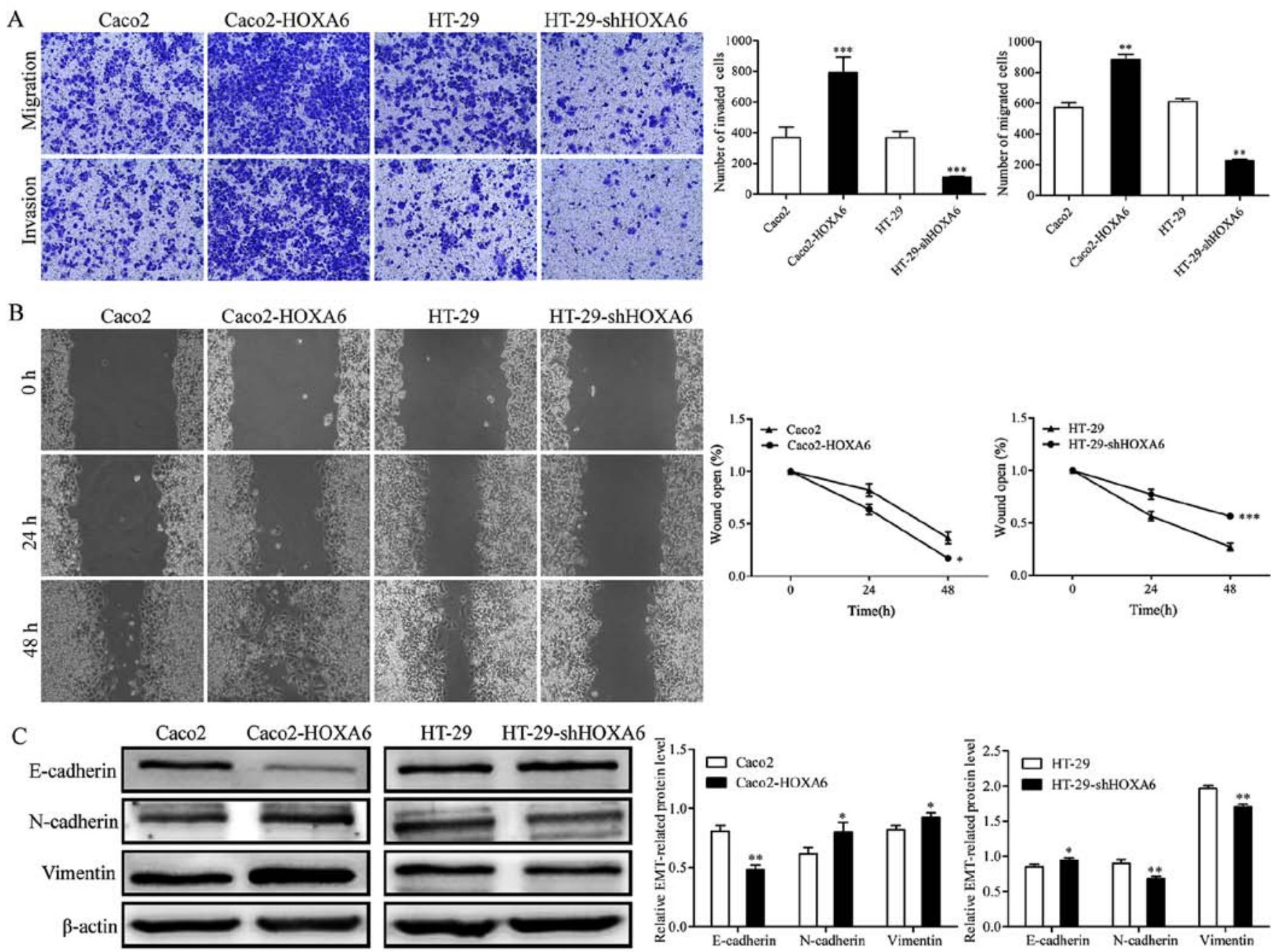

Figure 4. Migration and invasion of transfected cells as measured with Transwell and wound-healing assays, and the expression of EMT-related proteins. (A) Results of Transwell invasion and migration assays with the transfected cells (x100 magnification). (B) Results of a wound-healing assay to detect the migration of the transfected cells (x100 magnification). (C) Results of western blot analysis for EMT-related proteins, including E-cadherin, N-cadherin and Vimentin. Three independent experiments were performed for each assay. ${ }^{*} \mathrm{P}<0.05,{ }^{* * *} \mathrm{P}<0.01$ and ${ }^{* * * *} \mathrm{P}<0.001$. EMT, epithelial-mesenchymal transition; sh, short hairpin RNA; HOXA6, homeobox A6.

The findings of the present study suggested that HOXA6 was associated with the proliferation, apoptosis, migration and invasion of CRC cells, and further elucidated the potential mechanisms by which HOXA6 inhibited apoptosis and promoted migration and invasion. However, there were number of shortcomings in the present study and the potential mechanism underlying the regulation of cell proliferation requires further investigation. The number of clinical samples was insufficient, and additional samples are required to verify the expression of HOXA6 in colorectal tumors. There was also a lack of relevant clinical information regarding the study participants. In addition, HOXA6, as a transcription factor, mediates its biological effect by regulating the expression of downstream genes, which may provide a direction for further investigations.

In conclusion, the expression of HOXA6 was demonstrated to be associated with the proliferation, apoptosis, migration and invasion of CRC cells through experiments involving HOXA6 knockdown or transfection. These results suggest that HOXA6 is involved in the regulation of CRC, which may inform the development of strategies for the diagnosis and treatment of CRC.

\section{Acknowledgements}

The authors would like to thank Dr Tingxiu Xiang and the Chongqing Key Laboratory of Molecular Oncology and Epigenetics for providing the equipment, materials and technical supports.

\section{Funding}

No funding was received.

\section{Availability of data and materials}

The datasets used and/or analyzed during the current study are available from the corresponding author on reasonable request.

\section{Authors' contributions}

SW and FW made substantial contributions to the study conception, design, acquisition of data, analysis and interpretation of the data and were involved in the drafting of the 
manuscript. ZJ was involved in revising the manuscript critically for important intellectual content. All authors have read and approved the final manuscript.

\section{Ethics approval and consent to participate}

Informed consent was signed by all patients, and ethics approval was obtained from the Ethics Committee of the First Affiliated Hospital of Chongqing Medical University.

\section{Consent for publication}

Not applicable.

\section{Competing interests}

The authors declare that they have no competing interests.

\section{References}

1. Siegel RL, Miller KD, Fedewa SA, Ahnen DJ, Meester RG, Barzi A and Jemal A: Colorectal cancer statistics, 2017. CA Cancer J Clin 67: 177-193, 2017.

2. Siegel RL, Miller KD and Jemal A: Cancer Statistics, 2017. CA Cancer J Clin 67: 7-30, 2017.

3. Chen W, Zheng R, Baade PD, Zhang S, Zeng H, Bray F, Jemal A, Yu XQ and He J: Cancer statistics in China, 2015. CA Cancer J Clin 66: 115-132, 2016.

4. Wu S, Wu F and Jiang Z: Identification of hub genes, key miRNAs and potential molecular mechanisms of colorectal cancer. Oncol Rep 38: 2043-2050, 2017

5. Mourtzikou A, Stamouli M, Kroupis C, Christodoulou S, Skondra M, Kastania A, Pectasides D, Athanasas G and Dimas C: Evaluation of carcinoembryonic antigen (CEA), epidermal growth factor receptor (EGFR), epithelial cell adhesion molecule EpCAM (GA733-2), and carbohydrate antigen 19-9 (CA 19-9) levels in colorectal cancer patients and correlation with clinicopathological characteristics. Clin Lab 58: 441-448, 2012.

6. Shah N and Sukumar S: The Hox genes and their roles in oncogenesis. Nat Rev Cancer 10: 361-371, 2010.

7. Bhatlekar S, Fields JZ and Boman BM: HOX genes and their role in the development of human cancers. J Mol Med (Berl) 92 811-823, 2014

8. Takahashi O, Hamada J, Abe M, Hata S, Asano T, Takahashi Y, Tada M, Miyamoto M, Kondo S and Moriuchi T: Dysregulated expression of HOX and ParaHOX genes in human esophageal squamous cell carcinoma. Oncol Rep 17: 753-760, 2007.

9. Lv J, Cao XF, Ji L, Zhu B, Wang DD, Tao L and Li SQ: Association of $\beta$-catenin, Wnt 1 , Smad4, Hoxa9, and Bmi- 1 with the prognosis of esophageal squamous cell carcinoma. Med Oncol 29: 151-160, 2012.

10. Gu ZD, Shen LY, Wang H, Chen XM, Li Y, Ning T and Chen KN: HOXA13 promotes cancer cell growth and predicts poor survival of patients with esophageal squamous cell carcinoma. Cancer Res 69: 4969-4973, 2009.

11. Han Y, Lu S, Wen YG, Yu FD, Zhu XW, Qiu GQ, Tang HM, Peng ZH and Zhou CZ: Overexpression of HOXA10 promotes gastric cancer cells proliferation and HOXA10(+)/CD44(+) is potential prognostic biomarker for gastric cancer. Eur J Cell Biol 94: 642-652, 2015.

12. Sentani K, Oue N, Naito Y, Sakamoto N, Anami K, Oo HZ, Uraoka N, Aoyagi K, Sasaki H and Yasui W: Upregulation of HOXA10 in gastric cancer with the intestinal mucin phenotype: Reduction during tumor progression and favorable prognosis Carcinogenesis 33: 1081-1088, 2012.
13. He YX, Song XH, Zhao ZY and Zhao H: HOXA13 upregulation in gastric cancer is associated with enhanced cancer cell invasion and epithelial-to-mesenchymal transition. Eur Rev Med Pharmacol Sci 21: 258-265, 2017.

14. He X, Liu Z, Xia Y, Xu J, Lv G, Wang L, Ma T, Jiang L, Mou Y, Jiang X, et al: HOXB7 overexpression promotes cell proliferation and correlates with poor prognosis in gastric cancer patients by inducing expression of both AKT and MARKs. Oncotarget 8: 1247-1261, 2017

15. Joo MK, Park JJ, Yoo HS, Lee BJ, Chun HJ, Lee SW and Bak YT: The roles of HOXB7 in promoting migration, invasion, and anti-apoptosis in gastric cancer. J Gastroenterol Hepatol 31: 1717-1726, 2016.

16. Chen SW, Zhang Q, Xu ZF, Wang HP, Shi Y, Xu F, Zhang WJ, Wang P and Li Y: HOXC6 promotes gastric cancer cell invasion by upregulating the expression of MMP9. Mol Med Rep 14: 3261-3268, 2016 .

17. Zhang Q, Jin XS, Yang ZY, Wei M, Liu BY and Gu QL: Upregulated Hoxc6 expression is associated with poor survival in gastric cancer patients. Neoplasma 60: 439-445, 2013.

18. Liao WT, Jiang D, Yuan J, Cui YM, Shi XW, Chen CM, Bian XW, Deng YJ and Ding YQ: HOXB7 as a prognostic factor and mediator of colorectal cancer progression. Clin Cancer Res 17: 3569-3578, 2011.

19. Guo YB, Shao YM, Chen J, Xu SB, Zhang XD, Wang MR and Liu HY: Effect of overexpression ofHOXgenes on its invasive tendency in cerebral glioma. Oncol Lett 11: 75-80, 2016.

20. Dickson GJ, Liberante FG, Kettyle LM, O'Hagan KA, Finnegan DP, Bullinger L, Geerts D, McMullin MF, Lappin TR, Mills KI, et al: HOXA/PBX3 knockdown impairs growth and sensitizes cytogenetically normal acute myeloid leukemia cells to chemotherapy. Haematologica 98: 1216-1225, 2013.

21. Cheng TL and Chang WT: Construction of simple and efficient DNA vector-based short hairpin RNA expression systems for specific gene silencing in mammalian cells. Methods Mol Biol 408: 223-241, 2007.

22. Livak KJ and Schmittgen TD: Analysis of relative gene expression data using real-time quantitative PCR and the 2(-Delta Delta C(T)) method. Methods 25: 402-408, 2001.

23. Fernald $\mathrm{K}$ and Kurokawa M: Evading apoptosis in cancer. Trends Cell Biol 23: 620-633, 2013.

24. Brentnall M, Rodriguez-Menocal L, De Guevara RL, Cepero E and Boise LH: caspase-9, caspase-3 and caspase-7 have distinct roles during intrinsic apoptosis. BMC Cell Biol 14: 32, 2013.

25. Zeren T, Inan S, Vatansever HS and Sayhan S: Significance of apoptosis related proteins on malignant transformation of ovarian tumors: A comparison between Bcl-2/Bax ratio and p53 immunoreactivity. Acta Histochem 116: 1251-1258, 2014.

26. Samarghandian S, Nezhad MA and Mohammadi G: Role of caspases, Bax and Bcl-2 in chrysin-induced apoptosis in the A549 human lung adenocarcinoma epithelial cells. Anticancer Agents Med Chem 14: 901-909, 2014.

27. Boulares AH, Yakovlev AG, Ivanova V, Stoica BA, Wang G, Iyer S and Smulson M: Role of poly(ADP-ribose) polymerase (PARP) cleavage in apoptosis. Caspase 3-resistant PARP mutant increases rates of apoptosis in transfected cells. J Biol Chem 274: 22932-22940, 1999.

28. Mullen P: PARP cleavage as a means of assessing apoptosis. Methods Mol Med 88: 171-181, 2004.

29. Nieto MA, Huang RY, Jackson RA and Thiery JP: Emt: 2016. Cell 166: 21-45, 2016.

30. Heerboth S, Housman G, Leary M, Longacre M, Byler S, Lapinska K, Willbanks A and Sarkar S: EMT and tumor metastasis. Clin Transl Med 4: 6, 2015. 